\title{
Efectos de la implementación de una Casa de Espera Materna en la Micro red de Salud Pichanaki
}

\author{
Effects of the implementation of a maternity resting home in the Health Network Pichanaki \\ Dassine Jibaja-Calvo ${ }^{1,2}$, Pablo Best-Bandenay ${ }^{2}$, Luis Vera-Linares ${ }^{1}$, Michelle Lozada-Urbano ${ }^{3,4}$
}

\section{RESUMEN}

Objetivos: Determinar los resultados de la Implementación de una casa de espera materna en la atención de partos institucionales en gestantes de alto riesgo obstétrico provenientes de la Microrred Pichanaki. Métodos: Investigación de tipo retrospectivo, descriptivo, de corte transversal. Se tienen dos grupos uno que participa de la Casa de espera materna, donde se pretende indagar los resultados de la implementación y un grupo sin Casa de espera materna. Los datos corresponden al periodo 2009-2010. Se trabajó con información de tipo secundario. Se aplicó un muestreo de tipo aleatorio, con afijación proporcional y un nivel de confianza del $95 \%$. Siendo el tamaño de muestra de 58 historias, distribuidas así: Puesto de Salud Las Palmas (39) y Puesto de Salud Huantinini (19). Resultados: Las características de las madres que asisten al Casa de espera materna, tienen en promedio entre 16 y 35 años, su casa queda a más de cuatro horas de distancia, tiene un periodo intergenésico mayor a 18 meses, no tiene antecedentes de muerte perinatal, un $20 \%$ ha tenido abortos, el $60 \%$ ha tenido más de cuatro controles, un tercio de ellas tiene un peso normal y tienen anemia. De todas las variables usadas para describir a la población, la variable procedencia de las usuarias (tiempo en horas para alcanzar el Casa de espera materna, mostró un $p<0,05$; lo que nos indica que existe una diferencia significativa con el tiempo en horas para alcanzar el Casa de espera materna siendo mayor el tiempo en el grupo sin Casa de espera materna. Así mismo, la variable distribución de la paridad, mostro un $p<0,05$, lo que nos indica que existe una diferencia de la distribución de paridad entre las gestantes del grupo con Casa de espera materna y sin Casa de espera materna, siendo mayor la paridad en el grupo con Casa de espera materna. Los partos fueron atendidos en su gran mayoría por obstetras (94,7\% sin CEM y $82,1 \%$ con CEM). Conclusiones: No se ha encontrado ninguna relación entre ambos grupos con respecto a la identificación de complicaciones obstétricas de causa directa como: los antecedentes gíneco-obstétricos, infecciones uterinas, enfermedades hipertensivas, alteración del trabajo de parto y otras complicaciones. Un alto porcentaje de las madres que pertenecen al Casa de espera materna, han tenido un parto eutócico. La probabilidad de tener un parto con riesgo es 6 veces más en el grupo que no asiste al Casa de espera materna. Más de la mitad ingresa con buen estado general y en su totalidad egresa satisfactoriamente.

Palabras clave: Casa de Espera Materna, gestante, parto, alto riesgo obstétrico, mortalidad materna.

\section{ABSTRACT}

Objectives: The objective was to determine the results of the implementation of Maternity Resting Home (MRH) in hospital births in pregnant high-risk obstetrics (HRO) from the Health Network Pichanaki. Methods: Research retrospective, descriptive, cross-sectional. Have two groups one who participates in the MRH which aims to investigate the results of implementation and a group without MRH. Data refer to 2009-2010. We worked with secondary type information. Random sampling rate, proportional allocation and a confidence level of $95 \%$ was applied. As the sample size of 58 stories and distributed as two groups, Las Palmas (39) and Huantinini (19). Results: The characteristics of the mother who attends MRH has averaged between 16 and 35 years, your home is more than four hours away, has a greater than 18 months intergenesic period, no history of perinatal death, $20 \%$ have had abortions, $60 \%$ had more than four controls, one third of them have a normal weight and have anemia. Of all the variables used to describe the population, the variable origin of users (time in hours to reach the MRH showed a $p$ $<0.05$, which indicates that there is a significant difference over time in hours to reach the MRH time being greater in the group without MRH. Also, the variable distribution of parity, showed $p<0.05$, which indicates that there is a difference in the distribution of parity among pregnant with $\mathrm{MRH}$ group without $\mathrm{MRH}$, being greater parity in the group with $\mathrm{MRH}$. The deliveries were attended mostly by midwives (94.7\% without $\mathrm{MRH}$ and $82.1 \%$ with $\mathrm{MRH}$ ). Conclusions: Could not find any relationship between the two groups regarding the identification of obstetric complications direct cause as: the gynecological and obstetric history, uterine infections, hypertensive disease, abnormal labor and other complications. A high percentage of mothers who belong to $\mathrm{MRH}$, have had a vaginal delivery. The probability of having a birth risk is 6 times in the group that did not attend the MRH. More than half admitted in good general condition, and fully graduated successfully

\section{Keywords: Maternity waiting home, Pregnancy, Parturition, high risk obstetrics, Maternal mortality}




\section{INTRODUCCIÓN}

La mortalidad materna es uno de los indicadores sanitarios que con mayor claridad evidencia la inequidad y la exclusión social, así como la inequidad de género para la toma de decisiones, el poco respeto a los derechos humanos y el limitado acceso a los servicios sociales (United Nations, 1992).

En el Perú, la razón de mortalidad materna ha ido disminuyendo. EI INEI, (2001), refiere 185 muertes maternas por cada 100000 nacidos vivos, mientras que los informes del INEI (2011) e INEI (2014) indica 93 muertes maternas por cada 100 mil nacidos vivos (UNPFA, 2014).

Reducir la mortalidad materna es una prioridad que se refleja en el hecho de que se haya incluido como una de las ocho metas de desarrollo del milenio. La mortalidad materna expresa las profundas desigualdades entre países, dentro de ellos, entre regiones, y las clases sociales. Y no solo nos enfrentamos a la muerte de cientos de miles de mujeres cada año, sino que tiene como uno de sus efectos la mortalidad de los niños de dichas mujeres.

Al igual que otros países de América Latina y el Caribe, el desafío de Perú para mejorar la salud materna y neonatal se enfrenta a las disparidades debido a la etnicidad, la geografía y la extrema pobreza. Parte del desafío consiste en adaptar los servicios sanitarios actuales a las costumbres de las comunidades que en la actualidad no reciben la suficiente atención.

La intervención que ha mostrado ser eficaz para el logro de la reducción de la mortalidad materna es la asistencia del parto por personal calificado, esta necesidad surge al fracaso de una serie de estrategias como el adiestramiento de parteras tradicionales y el enfoque de riesgo perinatal. Contar con un personal entrenado con capacidad para prevenir, detectar y manejar las complicaciones obstétricas más graves, junto con el equipo, medicinas y otros suministros esenciales es el factor más importante en la prevención de la mortalidad materna, este personal calificado es aquel prestador de servicios de salud que tiene habilidades y conocimientos para la correcta atención del embarazo y parto (WHO, UNICEF, UNFPA, and the World Bank, 2005).
Las Casas de Espera Materna (CEM) surgen como un servicio de hospedaje de bajo costo cogestionadas por la sociedad civil, orientada a facilitar el acceso de las gestantes a cuidados obstétricos esenciales reduciendo los riesgos derivados de la presentación inesperada de una complicación obstétrica en su hogar o un Establecimiento de Salud (EE.SS). Según el portal del Ministerio de salud (MINSA), se cuentan con 502 casas de espera materna a nivel nacional, (Ministerio de Salud, 2013).

En este trabajo se analizaron los efectos de la implementación de la CEM, identificando su relación con el incremento de la cobertura de partos institucionales, las complicaciones obstétricas y condiciones de parto de la gestante, para tal estudio se contó con datos provenientes de dos establecimientos de salud de nivel básico, uno de ellos dispone de una CEM y el otro no.

La importancia de ejecutar tal estudio es proporcionar información crítica y relevante sobre el desempeño de la CEM, a fin de evaluar su utilización y relación con la atención oportuna de gestantes en alto riesgo obstétrico, señalar alternativas complementarias para elevar el nivel de parto institucional y proponer recomendaciones para mejorar y ampliar la red de las CEMs. (Ministerio de Salud, Dirección General de Promoción de la Salud).

El enfoque actual para la reducción de la muerte materna considera el embarazo como un factor de riesgo para el desarrollo de complicaciones obstétricas, por lo tanto toda gestante debe tener una adecuada evaluación y seguimiento. Las causas más frecuentes de muerte materna son: hemorragia obstétrica enfermedad hipertensiva del embarazo, infecciones, aborto y alteraciones del trabajo de parto. La mayoría de las muertes maternas son prevenibles, para esto, es necesario un control materno-fetal adecuado y un parto institucional (Sánchez, 2012).

Las CEM, llamadas también "Mamawasi", se han construido sobre la base de los centros de salud y hospitales. La CEM está diseñada para parecerse a una típica casa indígena de un pueblo agrícola. A las futuras madres se les permite traer a miembros de la familia a las casas, lo que aumenta su confianza y su nivel de comodidad en el uso del servicio. La CEM, 
que operan bajo la gestión de una organización comunitaria, son un centro alternativo que provee albergue, alimentación y actividades educativas a mujeres embarazadas originarias de comunidades rurales pobres y alejadas de los servicios de salud, tanto antes del parto como después del mismo. La atención alimentaria brindada a las madres gestantes, se coordinan con instituciones locales y organizaciones sociales de base y familiares de las gestantes; asimismo, el gobierno local, también puede asumir junto con la comunidad y las familias la responsabilidad de la alimentación de las beneficiarias, este último constituye el bastión del óptimo funcionamiento y sostenibilidad. Por ello, las CEMs se sitúan estratégicamente cerca de las unidades de salud, de modo que se facilite trasladar a las mujeres allí para que accedan a los servicios de parto institucional y de cuidado del puerperio y del recién nacido. El objetivo de las CEM es garantizar el alojamiento, la promoción básica de la salud y referencia a las mujeres embarazadas procedentes de las comunidades de difícil acceso a los servicios de salud admitidas como ARO para la atención institucional del parto en las unidades de mayor complejidad del MINSA.

La hipótesis que planteamos fue: la atención de las casas de espera materna que prestan a las mujeres gestantes disminuye la mortalidad materna y perinatal (MMP).

El objetivo fue determinar los resultados de la Implementación de una casa de espera materna en la atención de partos institucionales en gestantes de alto riesgo obstétrico provenientes de la Microrred Pichanaki

\section{MATERIALES Y MÉTODOS}

\section{Diseño de estudio}

Investigación de tipo retrospectivo, descriptivo, de corte transversal. En este estudio se tiene un grupo que participa de la CEM donde se pretende indagar los resultados de la implementación y un grupo sin CEM -sin implementación de una casa de espera- en la Microrred de salud del Distrito de Pichanaki. Los datos corresponden al periodo 2009-2010. Se trabajará con información de tipo secundario.

\section{Población y muestra}

La Población está constituida por Historias clínicas y Hojas del Centro Latinoamericano de Perinatología (CLAP) de los partos institucionales de gestantes (262) con alto riesgo obstétrico (ARO) que provienen del Puesto de salud (P.S) Las Palmas (176) y P.S Huantinini (86) entre Julio del 2009 a Septiembre del 2010, de la Microrred de salud de Pichanaki.

Cálculo del tamaño de la muestra: Para el cálculo del tamaño muestral relacionada a las historias clínicas de la población en estudio, se utilizó la técnica estadística que corresponde a la fórmula que utiliza la varianza máxima de proporciones, y que nos permitirá obtener estimaciones con una confianza del $95 \%$ y con un error máximo permisible deseado, cuya fórmula es la siguiente:

$$
n=\frac{n_{0}}{1+\frac{n_{0}}{N}} \quad n_{0}=\frac{N^{*} z_{\alpha / 2}^{2} p(1-p)}{d 2^{*}(N-1)+z_{\alpha / 2}^{2} * p(1-p)}
$$

Dónde:

$\mathrm{n}=$ Es el tamaño de la muestra.

$\mathrm{N}=$ Es el tamaño de la población

$Z^{\alpha / 2}=$ Nivel de Confianza

$p=$ Probabilidad del parámetro a evaluar (Considerando la Varianza Máxima con el fin de tener una muestra más precisa)

$\mathrm{d}=$ Error que se prevé cometer.

Para nuestra investigación tenemos que:

$\mathrm{N}=262$ historias clínicas

$Z=1,96$ considerando un nivel de confianza del $95 \%$

$\mathrm{p} \stackrel{\alpha / 2}{=} 0,0$

$d=0,05$ 
Reemplazando en la fórmula, tenemos:

$$
n_{0}=\frac{262 * 1,96^{2} * 0,5 *(1-0,5)}{0,05^{2} *(262-1)+1,96^{2} * 0,5(1-0,5)}=75
$$

Como $\mathrm{N}<\mathrm{n}_{0}\left(\mathrm{n}_{0}-1\right)$, entonces calculamos $\mathrm{n}$ de la siguiente manera:

$$
n=\frac{75}{1+\frac{75}{262}}=58
$$

La muestra se obtuvo mediante el muestreo de tipo aleatorio, con afijación proporcional y un nivel de confianza del $95 \%$. Siendo el tamaño de muestra para la presente investigación de 58 historias, distribuidas así: P.S Las Palmas (39) y P.S Huantinini (19).

Se elaboró una ficha para recolectar la información del control prenatal de las historias clínicas y hojas CLAP seleccionadas para la muestra, las que fueron diligenciadas por el personal técnico de la Microrred de Salud de Pichanaki, la misma que incluyó tres componentes de variables agrupadas: datos socio-demográficos y biológicos (edad, talla y procedencia), datos Gíneco- obstétricos y condiciones de parto, (estado de salud al ingreso y egreso de las gestantes en ARO referidas a la Microrred, medido en términos de bueno, regular y mal estado general de salud, así mismo la evaluación de la severidad de las complicaciones obstétricas de causa directa de las pacientes), datos de las gestantes de los registros de atención prenatal complementarias (hoja CLAP), y de las características de los partos institucionales.

Se hizo uso del análisis bivariado para presentar información referente a variables que tienen cierta relación. Se utilizaron las tablas de contingencia para identificar el tipo y el grado de complicaciones obstétricas de causa directa: antecedentes ginecoobstétricos, hemorragias obstétricas, infecciones uterinas, enfermedades hipertensivas del embarazo, alteraciones en el trabajo de parto; y la probabilidad de tener un parto con riesgo obstétrico en gestantes en la atención de partos institucionales en el grupo con CEM y sin CEM, se tendrá en cuenta las siguientes variables: Edad, talla, procedencia en horas al EESS, paridad, peso, periodo intergenésico, antecedentes de muerte perinatal - infantil, antecedentes patológicos personales, antecedentes Gineco-obstétricos, tiempo de embarazo, número de controles prenatales (CPN), ganancia de peso promedio durante la gestación, estado nutricional, personal que atendió el parto, estado de salud al ingreso y egreso, diagnóstico de salud al ingreso y egreso; los cuales calcularemos a través de indicadores (Olivera, Castelo y Cáceres, 2003).

Para identificar las complicaciones obstétricas de causa directa en las gestantes transferidas de la casa de espera materna, se observarán las siguientes variables para cada madre: Hemorragias obstétricas, infecciones uterinas y enfermedades hipertensivas del embarazo las mismas que también serán calculadas a través del uso de indicadores.

El presente estudio puede ser catalogado exento de supervisión parcial o completa, al no trabajar directamente con pacientes. Se solicitó autorizaciones correspondientes al área operativa y a la Dirección General de la Microrred, de donde se obtuvo las historias correspondientes para el desarrollo del estudio.

\section{RESULTADOS}

Según los resultados obtenidos del análisis de la base de datos generada de las historias clínicas, en la Tabla 1 , se observa que tanto para los grupos con CEM y sin CEM, existe mayor porcentaje de madres entre las edades de 16 a 35 años. El porcentaje para quienes viven a más de 4 horas para alcanzar el CEM, es mayor para el grupo sin CEM. En cuanto a la distribución de paridad, las multíparas son un $63,2 \%$ para el grupo con CEM. El $39 \%$ de madres tiene un periodo intergenésico mayor a 18 meses para el grupo con CEM. 
De todas las variables usadas para describir a la población, la variable procedencia de las usuarias (tiempo en horas para alcanzar el CEM, mostró un $p<0.05$; lo que nos indica que existe una diferencia significativa con el tiempo en horas para alcanzar el CEM siendo mayor el tiempo en el grupo sin CEM.
Así mismo, la variable distribución de la paridad, mostró un $p<0.05$, lo que nos indica que existe una diferencia de la distribución de paridad entre las gestantes del grupo con CEM y sin CEM, siendo mayor la paridad en el grupo con CEM.

Tabla 1. Características de las pacientes que participan en el estudio casas de espera materna CEM.

\begin{tabular}{|c|c|c|c|c|}
\hline & $\begin{array}{c}\text { Con CEM } \\
(n=39) \\
\%\end{array}$ & $\begin{array}{c}\text { Sin CEM } \\
(n=19) \\
\%\end{array}$ & $\begin{array}{l}\text { Prueba } \\
\text { Chi } \\
\text { Cuadrado }\end{array}$ & $\begin{array}{c}\text { Valor } \\
\mathbf{P}\end{array}$ \\
\hline \multicolumn{5}{|c|}{ Distribución de la edad } \\
\hline Menores de $16 a$ & 10,5 & 10,0 & 0,458 & 0,795 \\
\hline De 16 a $35 a$ & 84,2 & 80,0 & & \\
\hline Mayores de $35 a$ & 5,3 & 10,0 & & \\
\hline \multicolumn{5}{|c|}{ Distribución de la talla } \\
\hline Menor de $146 \mathrm{~cm}$ & 50,0 & 40,0 & 0,527 & 0,468 \\
\hline Mayor de $146 \mathrm{~cm}$ & 50,0 & 60,0 & & \\
\hline \multicolumn{5}{|c|}{ Procedencia de las usuarias (tiempo en horas para alcanzar el CEM) } \\
\hline Menos de $1 \mathrm{~h}$ & 39,5 & 5,0 & 15,45 & 0,001 \\
\hline De 1 a $2 \mathrm{~h}$ & 13,2 & - & & \\
\hline De 3 a $4 h$ & 10,5 & 5,0 & & \\
\hline Mayor de $4 \mathrm{~h}$ & 36,8 & 90,0 & & \\
\hline \multicolumn{5}{|c|}{ Distribución de la Paridad } \\
\hline Primipara & 26,3 & 45,0 & 8,09 & 0,017 \\
\hline Multipara & 63,2 & 25,0 & & \\
\hline Gran multipara & 10,5 & 30,0 & & \\
\hline \multicolumn{5}{|c|}{ Distribución del peso } \\
\hline Hasta 49 & 44,7 & 55,0 & 2,95 & 0,399 \\
\hline 50 a 59 & 44,7 & 35,0 & & \\
\hline 60 a 69 & 10,6 & 5,0 & & \\
\hline De 70 a más & - & 5,0 & & \\
\hline \multicolumn{5}{|c|}{ Periodo Intergenésico } \\
\hline Menor de $18 \mathrm{~cm}$ & 34,2 & 30,0 & 2,263 & 0,322 \\
\hline Mayor de $18 \mathrm{~cm}$ & 39,5 & 25,0 & & \\
\hline No aplica & 26,3 & 45,0 & & \\
\hline
\end{tabular}

El $66,7 \%$ de las gestantes del grupo con CEM no tienen antecedentes de muerte perinatalinfantil y solo el $5,1 \%$ cuenta con antecedentes; mientras que en el grupo sin CEM el $8 \%$ de las gestantes no tiene antecedentes de muerte perinatal-infantil y el $15,8 \%$ si cuenta con antecedentes.

El $41 \%$ de las gestantes del grupo con CEM no tienen antecedentes gíneco obstétricos, seguido de un $20,5 \%$ de gestantes que han tenido abortos. En el grupo sin CEM, el 36,8\% no tiene antecedentes gíneco-obstétricos, seguido de un $15,8 \%$ de gestantes que han tenido abortos.

El tiempo de embarazo fue de 37 a 42 semanas para el $92,3 \%$ de las gestantes del grupo con CEM, en contraste con el grupo sin CEM que fue del $100 \%$. El $73,3 \%$ de las gestantes del grupo sin CEM realizó más de 4 controles prenatales, mientras que en el grupo con CEM solo fue del $61,5 \%$ de gestantes.

El 92,3\% de las gestantes del grupo con CEM tuvieron un peso promedio durante la gestación considerado como "normal", mientras que en el grupo sin CEM las gestantes con peso promedio normal durante la gestación fue del $89,5 \%$. El $10,6 \%$ de las gestantes del grupo sin CEM tuvieron sobrepeso u obesidad durante la gestación, en contraste con el grupo con CEM que tuvo un $7,7 \%$ de gestantes con sobrepeso u obesidad.

En relación con la anemia, el $35,9 \%$ de gestantes del grupo con CEM tuvieron un $\mathrm{Hb}$ $<10$, mientras que en el grupo sin CEM fue del $42,1 \%$ quienes tienen un $\mathrm{Hb}<10$. 
Los partos fueron atendidos en su gran mayoría por Obstetras $(94,7 \%$ sin CEM y $82,1 \%$ con CEM).

En relación al estado de salud de las gestantes al ingreso del establecimiento, el $59 \%$ del grupo con CEM fue diagnosticado con aparente buen estado general, mientras que en el grupo sin CEM solo fue del $47,4 \%$. Con respecto al diagnóstico de salud al ingreso, se encontró que el $53,8 \%$ de gestantes del grupo con CEM tuvo fase latente, seguido de un $30,8 \%$ que tuvo fase activa, mientras que en el grupo sin CEM, el $57,9 \%$ tuvo fase latente y el $21,1 \%$ fase activa. Con respecto a los datos de la altura uterina $(A U)$ al final del embarazo expresado en centímetros, se encontró que el $76,9 \%$ de gestantes del grupo con CEM tuvo un feto que se desarrolló entre 30 a $35 \mathrm{~cm}$., seguido de un $12,8 \%$ que tuvo un desarrollo menor de $30 \mathrm{~cm}$, mientras que en el grupo sin CEM, el $84,2 \%$ tuvo un feto que se desarrolló entre 30 a $35 \mathrm{~cm}$. y el $10,5 \%$ tuvo un desarrollo menor de $30 \mathrm{~cm}$.

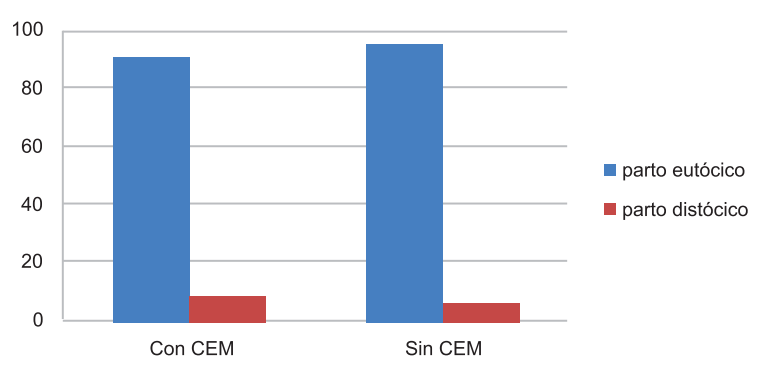

Figura 1. Total de gestantes de ambos grupos, son y sin CEM

En la Figura 1 se muestra el total de las gestantes de ambos grupos, todas tuvieron un aparente buen estado general de salud al egresar del establecimiento de salud.

Tabla 2. Tipo y el grado de riesgo obstétrico en gestantes en la atención de partos institucionales en el grupo con CEM y sin CEM

\begin{tabular}{|c|c|c|c|c|}
\hline Variables & $\begin{array}{c}\text { Con CEM } \\
(n=39) \\
\%\end{array}$ & $\begin{array}{c}\text { Sin CEM } \\
(n=19) \\
\%\end{array}$ & $\begin{array}{c}\text { Prueba } \\
\text { Chi } \\
\text { Cuadrado }\end{array}$ & $\begin{array}{l}\text { Valor } \\
P\end{array}$ \\
\hline \multicolumn{5}{|c|}{ Antecedentes de muerte perinatal - infantil } \\
\hline con antecedentes & $2(5,11)$ & $3(15,8)$ & 1,553 & 0,46 \\
\hline sin antecedentes & $26(66,7)$ & $8(42,1)$ & & \\
\hline No aplica & $11(28,6)$ & $8(42,1)$ & & \\
\hline \multicolumn{5}{|c|}{ Antecedentes Gineco-obstétricos } \\
\hline Abortos & $8(20,5)$ & $3(15,8)$ & 2,64 & 0,45 \\
\hline Otros & $3(7,7)$ & - & & \\
\hline Ninguno & $16(41)$ & $7(36,8)$ & & \\
\hline No aplica & $12(30,8)$ & $9(47,4)$ & & \\
\hline \multicolumn{5}{|l|}{ Tiempo de embarazo } \\
\hline Menor de 37 Ss & $3(7,7)$ & - & 1,541 & 0,214 \\
\hline De 37 a 42 Ss & $36(92,3)$ & $19(100)$ & & \\
\hline Mayor de 42 Ss & - & - & & \\
\hline \multicolumn{5}{|c|}{ Número de Controles Prenatales } \\
\hline Menor de 4 & $11(28,2)$ & $2(10,5)$ & 2,393 & 0,302 \\
\hline Mayor de 4 & $24(61,5)$ & $14(73,7)$ & & \\
\hline CPN completos & $4(10,3)$ & $3(15,8)$ & & \\
\hline \multicolumn{5}{|c|}{$\begin{array}{l}\text { Peso promedio durante la gestación, estado nutricional } \\
\text { Bajo }\end{array}$} \\
\hline Normal & $36(92,3)$ & $17(89,5)$ & 0,282 & 0,869 \\
\hline Sobrepeso & $2(5,1)$ & $1(5,3)$ & & \\
\hline Obesidad & $1(2,6)$ & $1(5,3)$ & & \\
\hline \multicolumn{5}{|l|}{ Anemia } \\
\hline $\mathrm{Hb}<10$ & $14(35,9)$ & $8(42,1)$ & 0,645 & 0,724 \\
\hline $\mathrm{Hb}>10$ & $24(61,5)$ & $11(57,9)$ & & \\
\hline Non consignado & $1(2,6)$ & - & & \\
\hline \multicolumn{5}{|c|}{ Personal que atendió el parto } \\
\hline Médico & $6(15,4)$ & $1(5,3)$ & 1,303 & 0,254 \\
\hline Obstetra & $32(82,1)$ & $18(94,7)$ & & \\
\hline Perdidos & $1(2,6)$ & - & & \\
\hline \multicolumn{5}{|c|}{ Estado de salud al ingreso } \\
\hline ABEG & 23(59) & $9(47,4)$ & 1,394 & 0,498 \\
\hline AREG & $1(2,6)$ & - & & \\
\hline AMEG & - & - & & \\
\hline No consignado & $15(38,5)$ & $10(52,6)$ & & \\
\hline
\end{tabular}


Diagnóstico de salud al ingreso

No trabajo de parto

Prodromos

Fase latente

Fase activa

Otros

\section{AU final}

$<$ a $30 \mathrm{~cm}$

de 30 a $35 \mathrm{~cm}$

$>$ a $35 \mathrm{~cm}$

No aplica

\section{Estado general al egreso}

ABEG

AREG

AMEG

No consignado

\section{Diagnóstico de Egreso}

Parto Eutósico

Parto Distósico

Otras complicaciones
$2(5,1)$

$3(7,7)$

$21(53,8)$

$21(30,8)$

$1(2,6)$

$5(12,8)$

$30(76,9)$

$3(7,7)$

$1(2,6)$

$39(100)$

$35(89,7)$

$3(7,7)$

$1(2,6)$
$4(21,1)$

$11(57,9)$

$4(21,1)$

-

$2(10,5)$

$16(84,2)$

$1(5,3)$

-

19(100)

18(94,7)

$1(5,3)$

-

3,826

0,43

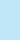

No se ha encontrado ninguna relación entre ambos grupos con respecto a la identificación de complicaciones obstétricas de causa directa como: los antecedentes gíneco-obstétricos, infecciones uterinas, enfermedades hipertensivas, alteración del trabajo de parto y otras complicaciones.

Tabla 3. Identificación de complicaciones obstétricas de causa directa en gestantes transferidas de la casa de espera materna.

\begin{tabular}{|c|c|c|c|c|}
\hline Variables & $\begin{array}{c}\text { Con CEM } \\
(\mathrm{n}=39) \\
\%\end{array}$ & $\begin{array}{c}\text { Sin CEM } \\
(n=19) \\
\%\end{array}$ & $\begin{array}{c}\text { Prueba } \\
\text { Chi } \\
\text { Cuadrado }\end{array}$ & $\begin{array}{c}\text { Valor } \\
\mathbf{P}\end{array}$ \\
\hline \multicolumn{5}{|l|}{ Antecedentes Gineco-obstétricos } \\
\hline Menor de $750 \mathrm{ml}$ & $1(2,56)$ & 0 & $0,496^{\mathrm{a}}$ & 0,48 \\
\hline No aplica & $38(97,44)$ & $19(100)$ & & \\
\hline \multicolumn{5}{|l|}{ Infecciones uterinas } \\
\hline No complicada & $6(15,38)$ & $3(15,79)$ & $0,002^{\mathrm{a}}$ & 0,97 \\
\hline No aplica & $33(84,6)$ & $16(84,21)$ & & \\
\hline \multicolumn{5}{|l|}{ Enfermedades hipertensivas } \\
\hline No aplica & 0 & 0 & & \\
\hline \multicolumn{5}{|l|}{ Alteración del trabajo de parto } \\
\hline Trabajo de parto prolongado & $1(2,56)$ & 0 & 0,54 & 0,763 \\
\hline $\begin{array}{l}\text { Incomp feto pélvica y estrechez pélvica } \\
\text { No aplica }\end{array}$ & $3(7,69)$ & 0 & & \\
\hline \multicolumn{5}{|l|}{ Otras complicaciones } \\
\hline Desgarro perineal & $2(5,12)$ & $1(5,26)$ & $0,133^{\mathrm{a}}$ & 0,94 \\
\hline Otros & $3(7,69)$ & $2(10,52)$ & & \\
\hline No aplica & $34(87,18)$ & $16(84,21)$ & & \\
\hline
\end{tabular}

No se ha encontrado ninguna relación entre ambos grupos con CEM y sin CEM con respecto a las condiciones de riesgo de parto. 
Tabla 4. Condiciones de riesgo y la influencia de la casa de espera materna (CEM).

\begin{tabular}{lccc}
\hline Variables & $\begin{array}{c}\text { Con CEM } \\
(\mathbf{n}=39) \\
\%\end{array}$ & $\begin{array}{c}\text { Sin CEM } \\
(\mathbf{n = 1 9 )} \\
\%\end{array}$ & OR \\
\hline Condiciones de riesgo de parto & & & 0,21 \\
$\mathrm{Si}$ & $13(33,33)$ & $4(21,1)$ & 3,97 \\
No & $25(64,1)$ & $15(78,9)$ & 0 \\
\hline
\end{tabular}

De la Tabla 4 se puede describir que hay 6 veces más probabilidad de tener un parto con riesgo en el grupo que no acude al CEM versus el grupo que si acude al CEM.

\section{DISCUSIÓN}

Los casos sin CEM, son menores a los de intervención con CEM, en todos los casos atendidos como partos institucionales de gestantes de ARO en las Palmas y Huantinini en la Micro red de Salud Pichanaki 2009- 2010.

Los casos que si presentan condiciones de riesgo, estuvieron en el grupo intervención con CEM.

Se ha encontrado información que evidencia que la atención prenatal institucional tendría utilidad para la ocurrencia de partos seguros. Tal es el estudio de la India (Bloom, 1999), ha revelado que, aquellas mujeres con un elevado nivel de atención prenatal tuvieron casi cuatro veces más posibilidades de ser asistidas durante el parto por personal competente $(\mathrm{OR}=$ $3,97$, IC $95 \%=1,9 ; 8,10)$, condición indispensable para recibir cuidados obstétricos esenciales en caso de requerirse. En este estudio se describe que hay seis veces más probabilidad de tener un parto con riesgo en el grupo que no acude al CEM versus el grupo que sí acude al CEM. La importancia de asistir a las Casas de Espera Maternas CEM es que incrementan el acceso a los servicios de salud ya que fueron diseñadas para enfrentar la inaccesibilidad geográfica de las mujeres embarazadas a los servicios de salud. En el estudio realizado por (Olivera, 2003) incluyo un grupo de gestantes inducidas a atención de parto institucional a través de una estrategia de salud, logrando la disminución de la mortalidad materna y perinatal.

Del mismo modo, un análisis de ENDES mostró que el riesgo de muerte materna era 9,82 $(8,75$ - 11,02) veces mayor en ausencia de atención prenatal. Aparentemente, el mecanismo explicativo es el mismo observado en la india. Más allá del valor predictivo de la atención prenatal, esta juega un papel importante en lograr que las gestantes elijan el parto institucional y, de esta manera, adquieran mayor protección en caso de aparecer complicaciones eventualmente mortales. EI riesgo de parto domiciliario en ausencia de atención prenatal es de $8,88(8,75-9,03)$ veces mayor, para este mismo estudio.

La creación de las casas de espera ha contribuido al incremento de los partos en las zonas rurales con asistencia de personal de salud capacitado (Cornale, 2009). Los partos con asistencia en el año 2000 eran solo del $20 \%$, cifra que subió al $48 \%$ en el 2007 (UNICEF, 2009).

La fuerte asociación positiva obtenida entre la asistencia al CEM de mujeres de ARO, el nivel de la atención obtenida durante el embarazo y el uso de la atención del parto seguro ayuda a explicar por qué el cuidado prenatal y durante el parto también podría estar asociado con una mortalidad materna reducida. Se recomienda continuar con la evaluación de las CEM para tener mayor información científica.

El $90 \%$ de las madres que pertenecen al CEM, han tenido un parto eutócico.

El pertenecer a un CEM da la probabilidad de tener un parto seis veces con menos riesgo, en comparación al grupo que no asiste al CEM y, en su totalidad egresa satisfactoriamente.

\section{REFERENCIAS BIBLIOGRÁFICAS}

Cornale G. (2009). Fondo de las Naciones Unidas para la Infancia. United Nations Plaza Nueva York, NY 10017, EEUU, pp $158 \quad 0$ b t e n i d o e n : http://ecodiario.eleconomista.es/salud/n 
oticias/1063990/02/09/Peru-redujo-lamortalidad-materna-a-casi-la-mitad-en40-anos.html\#.Kku8tugHv7DNagb

Ministerio de Salud. Dirección General de Promoción de la Salud. (sf). Guía de Implementación de Casas de Espera Materna con gestión comunal. http://www.bvsde.paho.org/bvsacd/cd51 /implementacion.pdf

Olivera, R., Castelo, JM. \& Cáceres, M. (2003). Hacia una maternidad rural segura. Universidad Nacional de San Antonio Abad del Cusco. SITUA, 12 (22), 11-15. ISSN versión electrónica 1609-726.

Instituto Nacional de Estadística e Informática (INEI). (2001). Encuesta Demográfica y de Salud Familiar 2000. Lima: INEI.

Instituto Nacional de Estadística e Informática. (INEI). (2011). Encuesta Demográfica y de Salud Familiar 2010. Lima: INEI. http://www.sis.gob.pe/portal/publicacion es/ExposicionENDES2011_20120523.p $\mathrm{df}$

Instituto Nacional de Estadística e Informática (INEI). (2014). Encuesta Demográfica de Salud Familiar ENDES 2013. Lima: INEI. http://www.inei.gob.pe/media/MenuRec ursivo/publicaciones_digitales/Est/Lib11 51/index.html.

Ministerio de Salud. (2013). Semana de la maternidad Saludable y Segura. Avances y Logros en la salud Materna. http://www.minsa.gob.pe/portada/Especi ales/2013/maternidad/presentacion.html

Fondo de Población de las Naciones Unidas (UNFPA) (2014). Reducción de la Mortalidad materna en el Perú. http://www.unfpa.org.pe/WebEspeciales
/2014/Jun2014/Ficha-MortalidadMaterna.pdf

UNICEF. (2009). EI Fondo de las Naciones Unidas para la Infancia (UNICEF) presentó en el Perú el Informe "Estado Mundial de la Infancia 2009". United Nations Plaza Nueva York, NY 10017, EEUU.

United Nations (1992). Department of Economics and Social Development, Population Division. World Population. New York: United Nations

WHO, UNICEF, UNFPA, and the World Bank. (2005). Maternal mortality in 2005 : estimates developed by WHO, UNICEF, UNFPA, and the World BanK. WHO Library Cataloguing-in-Publication Data. 2007, p 48.

Sánchez M. (2012). Recomendaciones generales para mejorar la calidad en la atención obstétrica. Comisión Nacional de arbitraje Médico. México DF.: Progreso. p 62.

http://www.conamed.gob.mx/prof_salud/ pdf/recom_obstetricia_web.pdf

Correo electrónico: dassine@yahoo.es

Revisado por pares:

Recibido: 16-03-2015

Aprobado: 20-06-2015 